\title{
Reseserch Suare \\ Ectopic gas in the fibular graft after anterior cervical corpectomy and fusion
}

Satoshi Nozawa ( $\nabla$ noza618@yahoo.co.jp )

Gifu University

Hiroki Kato

Gifu University

Masaya Kawaguchi

Gifu University

Asae Nozawa

Gifu University

Chizuo Iwai

Gifu University

Kazunari Fushimi

Gifu Prefectural General Medical Center

Kei Miyamoto

Gifu Municipal Hospital

Hideo Hosoe

Hirano general hospital

Katsuji Shimizu

Gifu Municipal Hospital

Masayuki Matsuo

Gifu University

Haruhiko Akiyama

Gifu University

Kazunari Yamada

Gifu University

\section{Research Article}

Keywords: ectopic, fusion, patients, bone, onset, follow, images, graft

Posted Date: August 5th, 2021

DOI: https://doi.org/10.21203/rs.3.rs-772502/v1 
License: (c) (i) This work is licensed under a Creative Commons Attribution 4.0 International License. Read Full License

Version of Record: A version of this preprint was published at BMC Musculoskeletal Disorders on November 29th, 2021. See the published version at https://doi.org/10.1186/s12891-021-04874-6. 


\section{Ectopic gas in the fibular graft after anterior cervical corpectomy and}

\section{fusion}

Satoshi Nozawa ${ }^{1}$, MD. PhD., Hiroki Kato ${ }^{2}$, MD. PhD., Masaya Kawaguchi² ${ }^{2}$ MD. PhD, Asae Nozawa ${ }^{2}$, MD. PhD., Kazunari Yamada ${ }^{1}$, MD. PhD., Chizuo Iwai ${ }^{1}$, MD. PhD., Kazunari Fushimi ${ }^{3}$, MD. PhD., Kei Miyamoto ${ }^{4}$, MD. PhD., Hideo Hosoe5, MD. PhD., Katsuji Shimizu, MD. PhD., Masayuki Matsuo 2 , MD. PhD., Haruhiko Akiyama ${ }^{1}$, MD. PhD.

1 Department of Orthopaedic Surgery, School of Medicine, Gifu University, Gifu, Japan

2 Department of Radiology, School of Medicine, Gifu University, Gifu, Japan

3 Department of Orthopedic Surgery, Gifu Prefectural General Medical Center, Gifu, Japan

4 Department of Orthopedic Surgery, Gifu Municipal Hospital, Gifu, Japan

5 Department of Orthopedic Surgery, Hirano General Hospital, Gifu, Japan 


\section{Corresponding Author:}

Satoshi Nozawa, M.D., Ph.D.

Department of Orthopedic Surgery

School of Medicine, Gifu University

1-1 Yanagido, Gifu city, Gifu 501-1194

Phone No.: 81-58-230-6333

Fax: 81-58-230-6334

Email address: noza618@yahoo.co.jp

The submitted manuscript does not contain information about medical device(s)/drug(s).

No funds were received in support of this work.

There were no relevant financial activities outside the submitted work. 
Title:

5

6 Ectopic gas in the fibular graft after anterior cervical corpectomy

7 and fusion

8 
10 Abstract

11 Ectopic gas in the graft is occasionally encountered upon follow-up computed tomography (CT) after anterior cervical corpectomy and fusion (ACCF). However, most cases lack inflammatory responses and manifestations of infection. We reviewed 112 patients who underwent $\mathrm{ACCF}$ and follow-up $\mathrm{CT}$, with a minimum follow-up period of 2 years. CT images were retrospectively reviewed to confirm the presence of ectopic gas in the graft and bone fusion. Bone fusion was defined as follows: mobility less than $2 \mathrm{~mm}$ between spinous processes on the flection-extension radiograph or a bone bridge on CT images. Of the 112 patients, 30 (27\%) patients had ectopic gas in the fibular grafts. Among them, ectopic gas was initially observed 3 months after surgery (early onset) in 23 (77\%) patients and 6 months after surgery (late onset) in the remaining seven $(23 \%)$ patients. Upon the latest follow-up CT, ectopic gas more frequently remained in late-onset $(4 / 7,57 \%)$ rather than in early-onset $(3 / 23,13 \%)$ cases $(p=$ 0.033). Bone fusion was not observed when CT images exhibited ectopic gas in the graft, whereas ectopic gas was not observed when CT images exhibited bone fusion. associated with pseudoarthrosis. 


\section{INTRODUCTION}

Anterior cervical corpectomy and fusion (ACCF) and strut bone grafting is an

established surgical option for the treatment of cervical spondylotic myelopathy (CSM) and ossification of the posterior longitudinal ligament (OPLL). As the strut bones, the ilium and fibula are routinely used to bridge the corpectomy defects. After such procedures, ACCF has been shown to achieve satisfactory clinical outcomes.

While its success in improving pain and disability scores has been documented ${ }^{1,2}$, one complication that remains a significant challenge is pseudarthrosis, which has been shown to occur at a rate of approximately $3-20 \%$ in in multilevel surgery ${ }^{3-5}$. Kuhns et al. stated that pseudarthrosis after anterior cervical discectomy and fusion (ACDF) has been recognized as a cause of continued cervical pain and unsatisfactory outcomes ${ }^{5}$, which can necessitate additional anterior or posterior surgery ${ }^{4-6}$.

Gas within the soft tissues usually indicates gas-producing infection caused by anaerobes bacteria or facultative gram-negative bacilli such as E. coli and Klebsiella spp. Ectopic gas in the graft is occasionally encountered upon follow-up computed tomography (CT) after ACCF, while the majority of cases lack inflammatory responses and manifestations of infection. Although the clinical significance of ectopic gas in the 
graft has not yet been established, to the best of our knowledge, no previous studies

47 have described ectopic gas in the graft after ACCF. The purpose of this study was to evaluate ectopic gas in the graft upon follow-up CT after ACCF and to determine the clinical significance from the perspective of pseudoarthrosis.

\section{MATERIALS AND METHODS}

This study was approved by the human research committee of the Institutional

Review Board of the Gifu University School of medicine and the requirement for

consent was waived because of the retrospective analysis. (Reference number 28-344)

All procedures involving human participants were in accordance with the 1964

Declaration of Helsinki and its later amendments. We retrospectively reviewed 112 cases (69 males; age range, 21-83 years; mean age, 59.0 years) that underwent ACCF for CSM, OPLL, and cervical disc herniation in our hospital between 2007 and 2018.

59 Patients were included if they had a minimum follow-up period of 3 years. The exclusion criteria included infection, tumor, cases without CT follow-up, and cases requiring reoperation. Bone graft reconstruction was performed using the fibula in 95 patients and the ilium in the remaining 17 patients. The follow-up period after ACCF ranged from 41-113 months (mean, 82 months). All patients underwent postoperative 
64 CT examinations 3, 6, 12, and 24 months after ACCF. White blood cell (WBC) counts,

65 C-reactive protein (CRP) levels, and the erythrocyte sedimentation rates (ESR) were

66 routinely measured during the follow-up period.

67

68 Operative Technique

69 After corpectomy, the length of the defect was measured, and the fibula or tricortical

70 ilium was harvested. During manual gentle traction of the skull, the graft was impacted

71 in the bone defect. In ACCF with 1- or 2-level corpectomies, anterior cervical plates

72 were indicated. The patients were immobilized with a Philadelphia collar until osseous

73 fusion was confirmed. In ACCF with 3-level corpectomies, patients were immobilized

74 with halo vests for 3 months, after that they wore Philadelphia collars until osseous

75 fusion was obtained.

$77 \quad$ CT imaging

Follow-up CT imaging was performed using an 8-slice CT system (LightSpeed

79 Ultra; GE Healthcare, Milwaukee, WI, USA), a 16-slice CT (LightSpeed Ultra 16; GE

80 Healthcare, Milwaukee, WI, USA), or a 64-slice CT system (Brilliance 64; Philips,

81 Best, The Netherlands). Unenhanced transverse and sagittal multiplanar reconstruction 
82 CT images were reconstructed with bone algorithms using a $2.5-\mathrm{mm}$ section thickness

83 and no overlap.

\section{Imaging assessment}

A radiologist with 21 years of post-training experience in musculoskeletal imaging and an orthopedic surgeon with 22 years of post-training experience in spine surgery, individually reviewed all CT images. Any disagreements between the reviewers were resolved by consensus.

First, the reviewers assessed the presence of ectopic gas in the graft upon follow-up

91 CT images. Approximately, a window level and window width were changed to clearly

92 identify ectopic gas using commercially available DICOM viewers. According to the timing of the initial appearance of ectopic gas, patients with ectopic gas were classified as early onset ( 3 months after surgery) and late onset (6 months after surgery).

95 Second, the reviewers also assessed the presence of the bone fusion of the graft.

96 Based on the findings from a previous $\operatorname{study}^{7}$, bone fusion of the graft was defined as

97 follows: mobility less than $2 \mathrm{~mm}$ between spinous processes on a flection-extension radiograph or a bone bridge on $\mathrm{CT}$ images. 


\section{Statistical analysis}

101 Statistical analysis was performed using SPSS version 22.0 (IBM Corp., Armonk,

102 NY, USA). Fisher's exact tests were used to compare the amount of the remaining

103 ectopic gas identified in the latest follow-up CT between early and late-onset cases. $P$ -

104 values $<0.05$ were considered statistically significant.

106 Result

107 Of the 112 included patients, $30(27 \%)$ patients had ectopic gas in the graft in the

108 follow-up CT images. All 30 bone grafts with ectopic gas were reconstructed using the

109 fibula. Four, 14, 10, and two cases had 1-, 2-, 3-, and 4-level corpectomy, respectively.

110 No signs of infection were found during the follow-up period in any of the patients.

111 Among 30 patients with ectopic gas, ectopic gas initially appeared 3 months after

112 surgery (early onset) in 23 patients and 6 months after surgery (late onset) in the

113 remaining seven patients. Upon the latest follow-up CT, ectopic gas disappeared in 20

114 (87\%) early-onset and three (43\%) late-onset cases, whereas ectopic gas remained in

115 three (13\%) early-onset and four (57\%) late-onset cases. Thus, ectopic gas more

116 frequently remained in the late-onset $(4 / 7,57 \%)$ than in the early-onset $(3 / 23,13 \%)$

117 cases $(p=0.033)$. 
Thirty cases with ectopic gas were classified into six groups based on the presence or

119 absence of ectopic gas (Figure 1). Among 23 early-onset cases, ectopic gas continued to

120 appear in one case (Group I, Figure 2), whereas it disappeared in 22 cases 6 months

121 after surgery. It continued to disappear in 17 cases (Group IV, Figure 5), while ectopic

122 gas appeared again in five cases 1-3 years after surgery. Among five cases with the

123 reappearance of ectopic gas, ectopic gas continued to remain in two cases (Group II,

124 Figure 3), whereas it disappeared again in three cases (Group III, Figure 4) upon the

125 latest follow-up CT images. Conversely, among seven late-onset cases, ectopic gas

126 continued to remain in four cases (Group V, Figure 6), whereas it disappeared in three

127 cases (Group VI, Figure 7) 1-3 years after surgery.

128 The relationship between bone fusion and ectopic gas in the graft upon the latest

129 follow-up CT is summarized in Table 1. Bone fusion was not observed when CT images

130 exhibited ectopic gas in the graft $(n=7)$, whereas ectopic gas was not observed when

131 CT images exhibited bone fusion $(n=97)$.

\section{Discussion}

134 ACCF is a reliable and effective procedure for treating CSM, OPLL, and cervical disc

135 herniation ${ }^{3,8-13}$. The advantage is that the spinal cord is directly decompressed by 
136 removing ossified lesions, and the anterior column is stabilized by the strut graft.

137 Although we occasionally encounter ectopic gas in the graft on CT images during the

138 postoperative course, no previous studies have reported ectopic gas in the graft after

139 ACCF. To investigate the clinical significance of this unknown imaging finding, we

140 reviewed 112 cases treated with ACCF. Ectopic gas in the fibular graft was observed at

141 both early and late onset after ACCF; late-onset gas tended to significantly persist. The

142 remaining gas was strongly associated with pseudoarthrosis. Pseudarthrosis after

143 ACDF or ACCF has been recognized as a cause of continued cervical pain and

144 unsatisfactory outcomes ${ }^{5}$, which can necessitate additional anterior or posterior

145 surgery $^{4-6}$. Based on the signs of pseudoarthrosis, we could give the patient some advice

146 such as prolonged brace wearing or restriction of neck range of motion.

147 The presence of ectopic gas in vessels in and around the spine, and within the skull,

148 usually leads to concerns regarding infections ${ }^{14}$. However, the presence of ectopic gas

149 does not always mean infections. The vacuum disc phenomenon is caused by an

150 accumulation of gas, principally nitrogen, within the crevices of the intervertebral discs

151 or adjacent vertebrae due to disc degeneration ${ }^{14-16}$. Intravertebral gas within a vertebral

152 compression fracture is typically nitrogen and it is caused by decreased pressure and

153 volume of the vertebra due to the ischemic vertebral collapse ${ }^{17}$. Extra and intradural gas 
154 may cause nerve root compression ${ }^{18,19}$. However, the etiology of ectopic gas in the

155 grafted bone is still unknown.

156 The accumulation of gas within the bone marrow is observed in various conditions

157 such as osteomyelitis, focal ischemia and osteonecrosis, posttraumatic states, and

158 solitary bone $\operatorname{cysts}^{20}$. Another rare condition associated with intraosseous gas includes

159 pneumatocyst, which is a benign cyst-like lesion that is filled with nitrogen within the

160 bone ${ }^{20-24}$. Pneumatocysts most frequently occur in the ilium and the sacrum ${ }^{24}$. The

161 transport of gas from the intervertebral disc into the adjacent subchondral bone via the

162 vertebral end plate may cause intraosseous gas ${ }^{25}$. Laufer et al. speculated that the gas

163 within the pneumatocyst is nitrogen released from the adjacent joints ${ }^{22}$.

164 Coulier et al. speculated the physiological algorithm for the vacuum

165 phenomenon ${ }^{19,26}$. They hypothesized that in permeable anatomic structures, a

166 sufficiently prolonged distraction allows for progressive penetration of a variable

167 mixture predominantly composed of gas or fluid depending on the nature of the

168 neighboring tissues. When distraction is reduced or compression occurs, gas and/or

169 fluid may be re-injected into the neighboring tissues or forced into blind spaces to form

170 predominantly gaseous or mixed hydro gaseous collections. Repetition of this

171 mechanism results in the "pumping" phenomenon ${ }^{19}$. 
174 for three disc levels fused were much higher than those for two disc levels fused ${ }^{27}$. Graft dislodgement was found in 20 of the 405 patients treated with ACCF in five studies ${ }^{8,28-}$

$176 \quad 31$.

177 We believe that the etiology of ectopic gas in the fibular graft after ACCF is similar

178 to that of the vacuum phenomenon or pneumatocyst. Based on our findings we

179 hypothesize, that gas in cases with early onset is caused by migration of air into the

180 graft during surgery, while gas in cases with late onset is caused by micromotion

181 between the graft and the vertebral body. The fact that ectopic gas disappeared after

182 graft fusion was achieved supports our theory.

183 Moreover, in this series, ectopic gas more frequently remained in cases with late

184 onset $(4 / 7,57 \%)$ than in those with early onset $(3 / 23,13 \%)(p=0.033)$. This suggests

185 that the presence of ectopic gas in the early postoperative stage could predict the gas

186 remaining at postoperative years $1-3$, which is related to the non-union of the graft.

\section{Conclusion}


190 Ectopic gas in the fibular graft was observed at both early and late onset after ACCF;

191 late-onset gas tended to significantly remain. The remaining gas was strongly associated

192 with pseudoarthrosis, therefore pseudoarthrosis should be considered when ectopic gas

193 in the graft is observed on CT images. The careful observation of ectopic gas in the graft

194 is a simple and useful method for predicting graft fusion.

195

196

197 
1991 Bohlman, H. H., Emery, S. E., Goodfellow, D. B. \& Jones, P. K. Robinson anterior cervical discectomy and arthrodesis for cervical radiculopathy. Long-term follow-up of one hundred and twenty-two patients. J Bone Joint Surg Am 75, 1298-1307, doi:10.2106/00004623-199309000-00005 (1993). Carette, S. \& Fehlings, M. G. Clinical practice. Cervical radiculopathy. $N$ Engl $J$ Med 353, 392-399, doi:10.1056/NEJMcp043887 (2005). Ikenaga, M., Shikata, J. \& Tanaka, C. Long-term results over 10 years of anterior corpectomy and fusion for multilevel cervical myelopathy. Spine 31, 1568-1574 (2006). Anterior Cervical Fusion: Case Series and Systematic Review. Global spine journal 10, 559-570, doi:10.1177/2192568219863808 (2020).

212 the treatment of cervical pseudarthrosis with posterior fusion. Spine (Phila Pa 1976) 30, 2424-2429, doi:10.1097/01.brs.0000184314.26543.7d (2005). Neo, M., Fujibayashi, S., Yoshida, M. \& Nakamura, T. Spinous process plate fixation as a salvage operation for failed anterior cervical fusion. Technical note. $J$ Neurosurg Spine 4, 78-81, doi:10.3171/spi.2006.4.1.78 (2006).

Cannada, L. K., Scherping, S. C., Yoo, J. U., Jones, P. K. \& Emery, S. E. Pseudoarthrosis of the cervical spine: a comparison of radiographic diagnostic measures. Spine (Phila Pa 1976) 28, 46-51, doi:10.1097/00007632-200301010-00012 (2003). decompression and arthrodesis for the treatment of cervical spondylotic myelopathy. Two to seventeen-year follow-up. J Bone Joint Surg Am 80, 941-951, doi:10.2106/00004623-199807000-00002 (1998).

Ikenaga, M., Shikata, J. \& Tanaka, C. Anterior corpectomy and fusion with fibular strut grafts for multilevel cervical myelopathy. J Neurosurg Spine 3, 79-85 (2005).

Komura, S. et al. Anterior cervical multilevel decompression and fusion using fibular strut as revision surgery for failed cervical laminoplasty. Archives of Orthopaedic and Trauma Surgery 131, 1177-1185, doi:10.1007/s00402-010-1248-3 (2011).

1 Komura, S., Miyamoto, K., Hosoe, H., Iinuma, N. \& Shimizu, K. Lower Incidence of Adjacent Segment Degeneration After Anterior Cervical Fusion Found With Those 

Clinical Spine Surgery 25, 23-29, doi:10.1097/BSD.0b013e31820bb1f8 (2012). for the treatment of oppressive myelopathy owing to cervical ossification of posterior longitudinal ligament: a meta-analysis. Eur Spine J27, 1375-1387, doi:10.1007/s00586-017-5451-6 (2018). Nozawa, S., Miyamoto, K., Sakaguchi, Y., Hosoe, H. \& Shimizu, K. Ossification of the posterior longitudinal ligament associated with rheumatoid arthritis. Orthopedics 27, 623-624 (2004). intracranial, and intraspinal ectopic gas (part III). Emergency radiology 24, 411416, doi:10.1007/s10140-017-1492-8 (2017). I., K. The VF in the Intervertebral Discs. Acta Radiologica 23, 173-179 (1942). W., M. Über die Bedingungen des Hersvortretens der Wirklichen Genlenkspalte auf den Röntgenbild. Acta Radiol 18, 733-741 (1937). Maldague, B. E., Noel, H. M. \& Malghem, J. J. The intravertebral vacuum cleft: a sign of ischemic vertebral collapse. Radiology 129, 23-29, doi:10.1148/129.1.23 (1978).

8 Ricca, G. F., Robertson, J. T. \& Hines, R. S. Nerve root compression by herniated intradiscal gas. Case report. J Neurosurg 72, 282-284, doi:10.3171/jns.1990.72.2.0282 (1990).

9 Coulier, B. The spectrum of vacuum phenomenon and gas in spine. JBR-BTR : organe de la Societe royale belge de radiologie $(S R B R)=$ orgaan van de Koninklijke Belgische Vereniging voor Radiologie (KBVR) 87, 9-16 (2004).

Yamamoto, T. et al. Natural course of an intraosseous pneumatocyst of the cervical spine. AJR Am J Roentgenol 179, 667-669, doi:10.2214/ajr.179.3.1790667 (2002).

Hoover, J. M., Wenger, D. E., Eckel, L. J. \& Krauss, W. E. Cervical pneumatocyst. $J$ Neurosurg Spine 15, 332-335, doi:10.3171/2011.5.Spine10627 (2011).

Laufer, L., Schulman, H. \& Hertzanu, Y. Vertebral pneumatocyst. A case report. Spine (Phila Pa 1976) 21, 389-391 (1996).

Matsukubo, Y. et al. Intravertebral pneumatocysts of the cervical spine. Neuroradiology 55, 1341-1344, doi:10.1007/s00234-013-1279-x (2013).

Hall, F. M. \& Turkel, D. Case report 526: Intraosseous pneumocyst of the ilium. Skeletal Radiol 18, 127-128 (1989).

5 Karasick, D. \& Eason, M. A. Vertebral pneumatocyst mimicking susceptibility artifact on MR imaging. AJR Am J Roentgenol 170, 221, 
doi:10.2214/ajr.170.1.9423641 (1998).

27026 Resnick, D., Niwayama, G., Guerra, J., Jr., Vint, V. \& Usselman, J. Spinal vacuum phenomena: anatomical study and review. Radiology 139, 341-348, doi:10.1148/radiology.139.2.7220878 (1981).

273

27 Jiang, S. D., Jiang, L. S. \& Dai, L. Y. Anterior cervical discectomy and fusion versus anterior cervical corpectomy and fusion for multilevel cervical spondylosis: a systematic review. Arch Orthop Trauma Surg 132, 155-161, doi:10.1007/s00402-0111402-6 (2012)

277

Hilibrand, A. S., Fye, M. A., Emery, S. E., Palumbo, M. A. \& Bohlman, H. H. Increased rate of arthrodesis with strut grafting after multilevel anterior cervical decompression. Spine (Phila Pa 1976) 27, 146-151, doi:10.1097/00007632200201150-00005 (2002).

281 Nirala, A. P., Husain, M. \& Vatsal, D. K. A retrospective study of multiple interbody grafting and long segment strut grafting following multilevel anterior cervical decompression. British journal of neurosurgery 18, 227-232, doi:10.1080/02688690410001732643 (2004).

$28530 \quad$ Yonenobu, K. et al. Choice of surgical treatment for multisegmental cervical spondylotic myelopathy. Spine (Phila Pa 1976) 10, 710-716, doi:10.1097/00007632198510000-00004 (1985). anterior cervical discectomy fusion and cervical corpectomy fusion using titanium cages for reconstruction: analysis of outcome and long-term follow-up. Eur Spine $J$ 18, 654-662, doi:10.1007/s00586-009-0897-9 (2009). 
296 S.N. and H.K. contributed to reporting the present manuscript. S.N., H.K., M.K., A.N. 297 and M.M. contributed to conception and design of the present study. K.Y., C.I., K.F., 298 K.M., H.H., K.S. and H.A. contributed to conducting the present study and to edit the 299 present manuscript. All authors reviewed and approved the final manuscript.

300

301

302

\section{Competing interests:}

303 The authors declare no competing interests.

304 
306 Fig. 1. Flowchart of the study population classified into six groups.

Fig. 2. A 72-year-old man underwent anterior cervical corpectomy and fusion due to cervical spondylotic myelopathy (Group I).

310 Two-level corpectomy and a fibula graft were performed. The computed tomography

311 image shows ectopic gas (arrow) in the fibular graft 3 months after surgery (Figure 2A).

312 The gas remained (arrow) without sufficient bone fusion 1 year after surgery (Figure 313 2B).

Fig. 3. A 65-year-old man underwent anterior cervical corpectomy and fusion due to cervical spondylotic myelopathy (Group II).

317 Three-level corpectomy and a fibula graft were performed. The computed tomography image shows ectopic gas (arrow) in the fibular graft 3 months after surgery (Figure 3A).

319 The gas disappeared 6 months after surgery (Figure 3B) but it appeared again (arrow) with bony erosion of the graft 1 year after surgery (Figure 3C). Three months after graft breakage, the gas (arrow) remained with bony erosion of the graft (Figure 3D). After additional posterior fusion, the gas disappeared as bone fusion was achieved (Figures 
Fig. 4. A 71-year-old woman underwent anterior cervical corpectomy and fusion due to cervical spondylotic myelopathy (Group III).

327 Two-level corpectomy and a fibula graft were performed. The computed tomography image showed ectopic gas (arrow) in the fibular graft 3 months after surgery (Figure 4A). The gas disappeared 6 months after surgery (Figure 4B) but it appeared again (arrow) without sufficient bone fusion 1 year after surgery (Figure 4C). The gas disappeared again with sufficient bone fusion 18 months after surgery (Figures 4D, E).

Fig. 5. A 54-year-old man underwent anterior cervical corpectomy and fusion due to cervical disc herniation (Group IV).

One-level corpectomy and a fibula graft were performed. The computed tomography image showed ectopic gas (arrow) in the fibular graft 3 months after surgery (Figure 5A). The gas disappeared 6 months after surgery (Figure 5B) and 2 years after surgery (Figure 5C) with sufficient bone fusion.

Fig. 6. An 82-year-old man underwent anterior cervical corpectomy and fusion due to 
341 cervical spondylotic myelopathy (Group V).

342 Two-level corpectomy and a fibula graft were performed. The computed tomography

343 image showed no ectopic gas (arrow) in the fibular graft 3 months after surgery (Figure

344 6A). The gas appeared 6 months after surgery (Figure 6B) and remained 20 months after

345 surgery (Figure 6C) without sufficient bone fusion.

346

347 Fig. 7. A 70-year-old man underwent anterior cervical corpectomy and fusion due to

348 cervical spondylotic myelopathy (Group VI).

349 Three-level corpectomy and a fibula graft were performed. The computed tomography

350 image showed no ectopic gas (arrow) in the fibular graft 3 months after surgery (Figure

351 7A). The gas appeared 6 months after surgery without sufficient bone fusion (Figure

352 7B) but disappeared 2 years after surgery with sufficient bone fusion (Figure 7C).

353 
Table 1. Relationship between bone fusion and ectopic gas in the graft on the latest follow-up CT

Bone fusion + Bone fusion -

$$
(n=97) \quad(n=15)
$$

$\begin{array}{lrr}\text { Ectopic gas }-(n=105) & 97 & 8 \\ \text { Ectopic gas }+(n=7) & 0 & 7\end{array}$

Values are given as $n$ 
Figure1

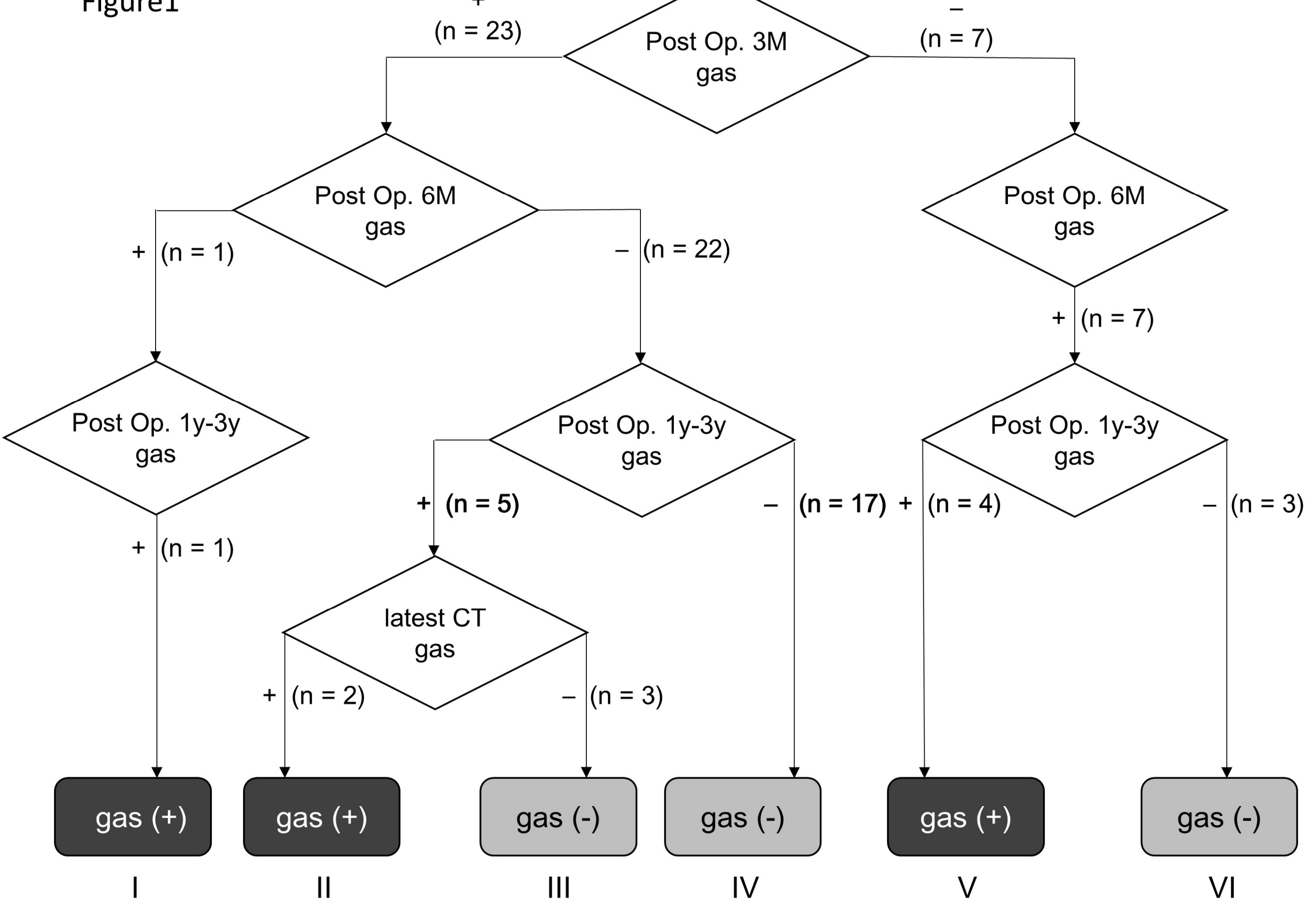



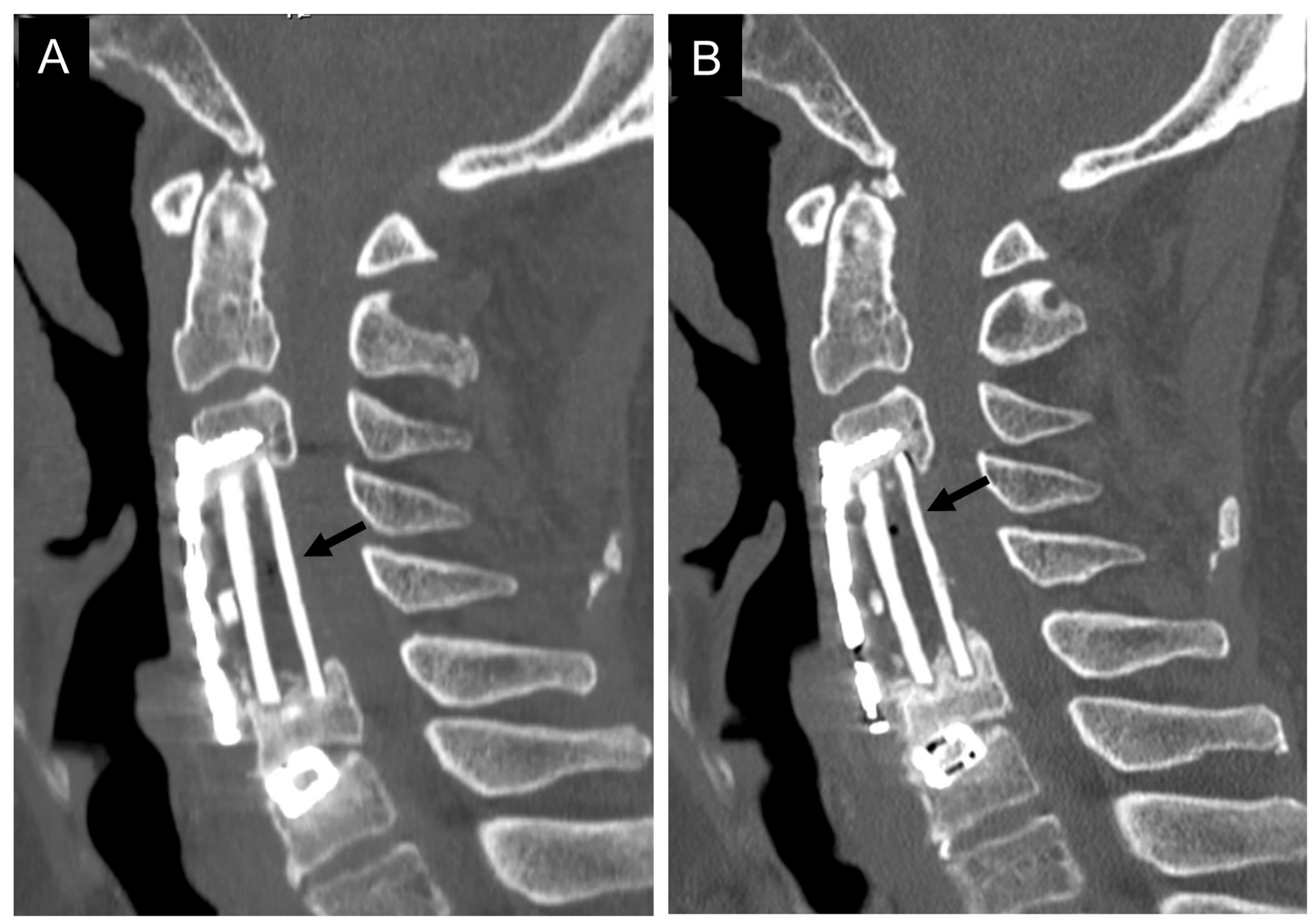

Figure 2 


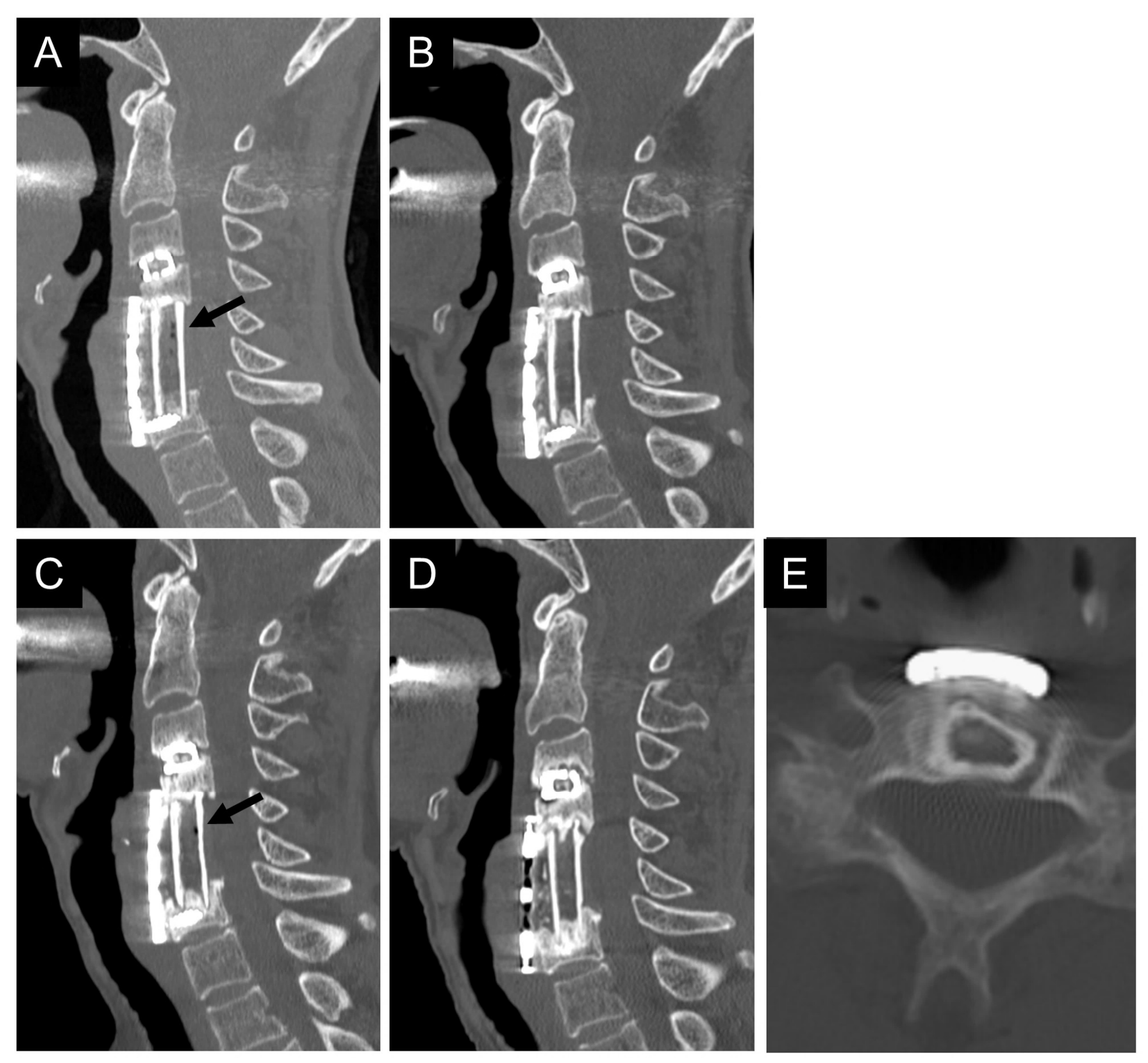

Figure 4 


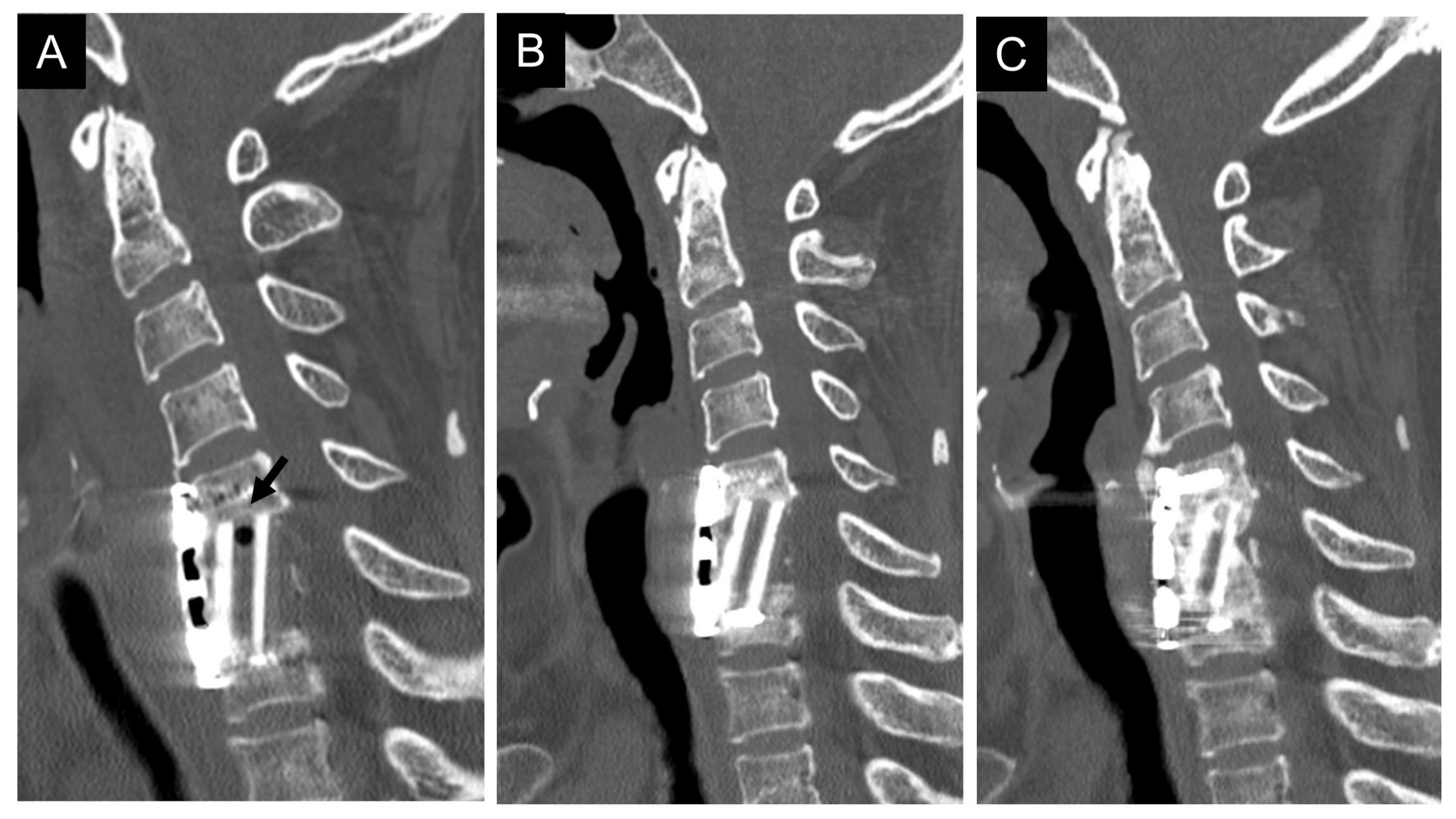

Figure 5 


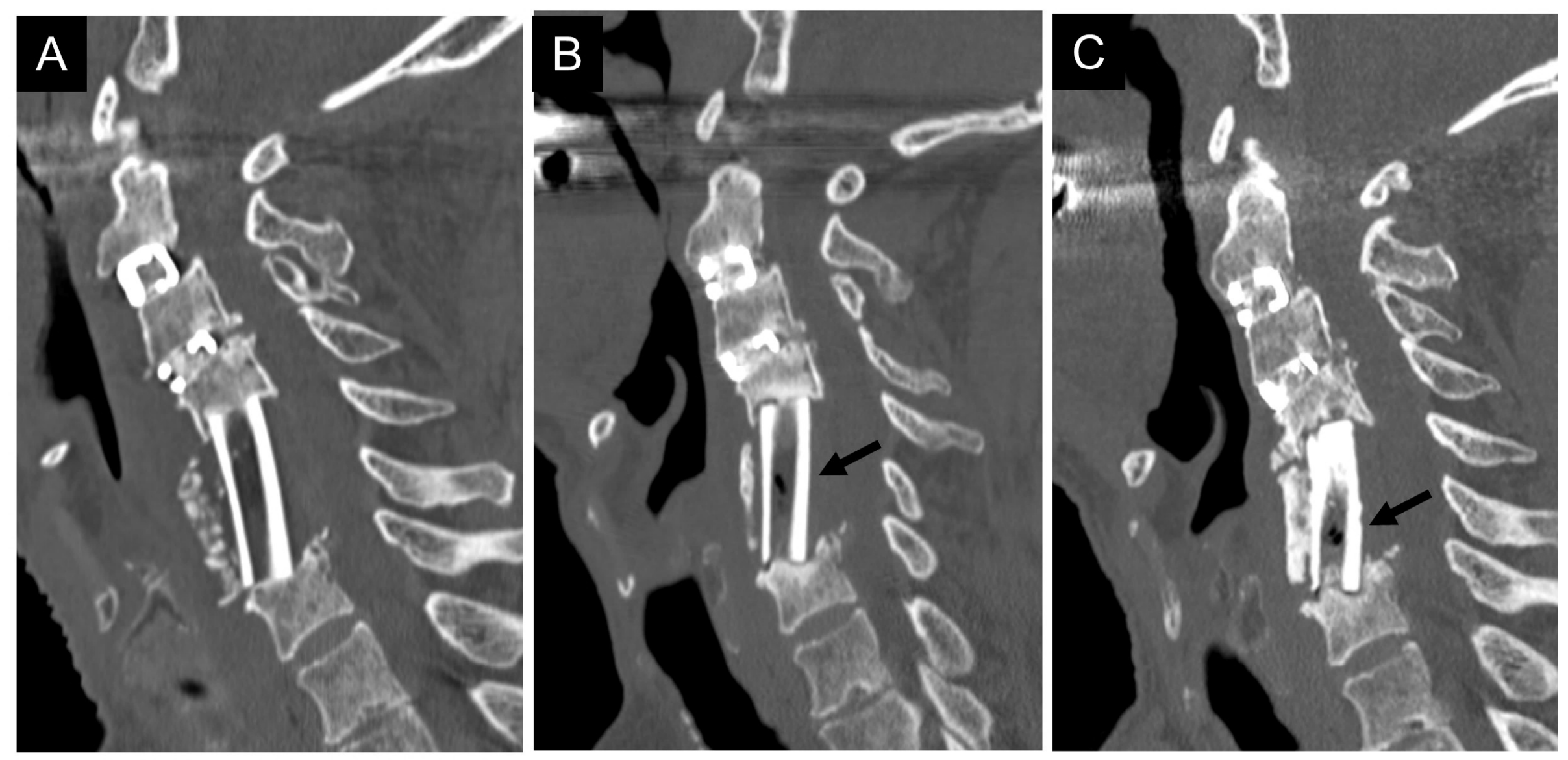

Figure 6 


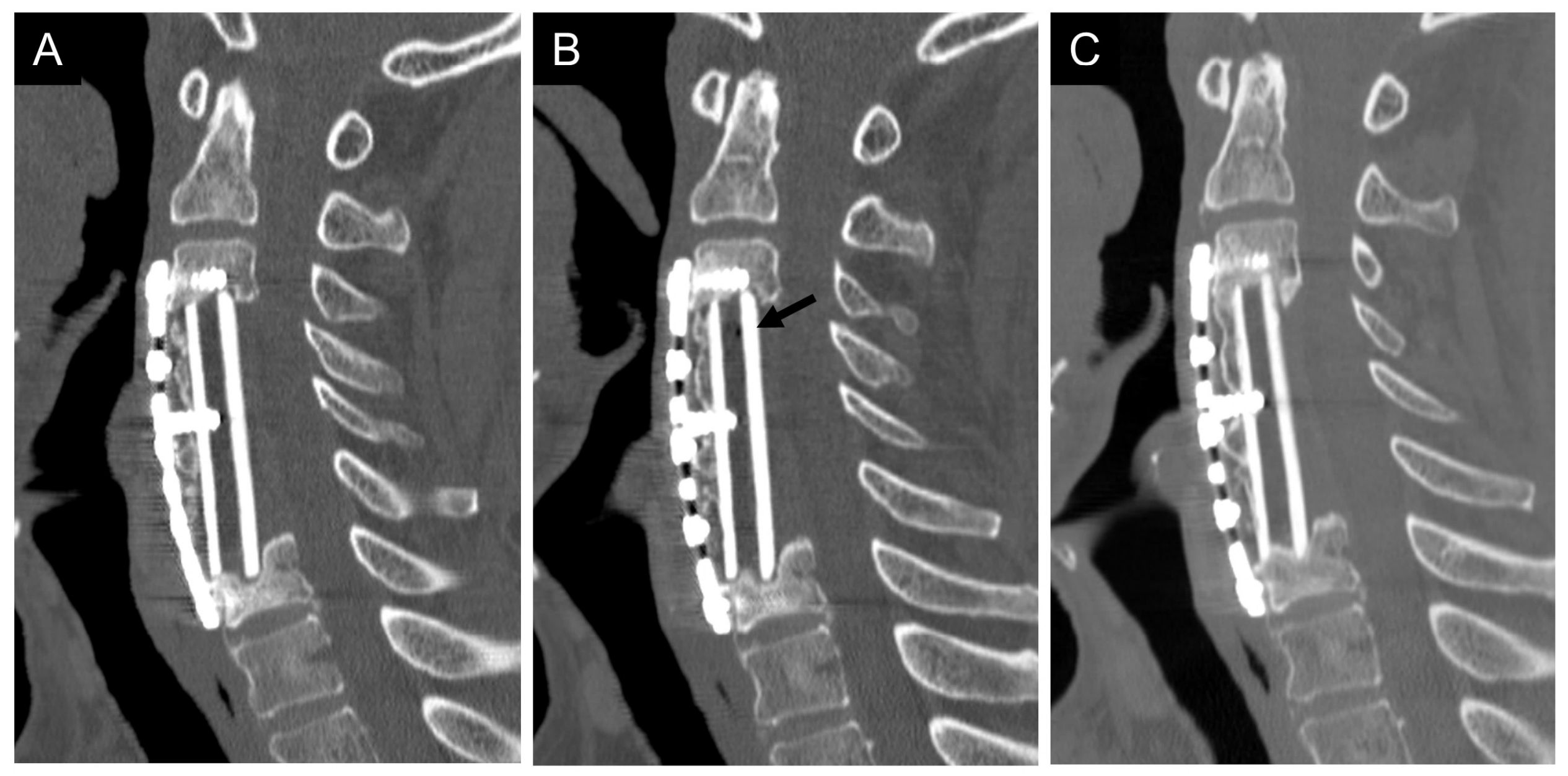

Figure 7 